\title{
Regularity and $h$-polynomials of edge ideals
}

\author{
Takayuki Hibi \\ Department of Pure and Applied Mathematics \\ Graduate School of Information Science and Technology \\ Osaka University \\ Suita, Osaka, 565-0871, Japan \\ hibi@math.sci.osaka-u.ac.jp
}

\author{
Kazunori Matsuda \\ Kitami Institute of Technology \\ Kitami, Hokkaido 090-8507, Japan \\ kaz-matsuda@mail.kitami-it.ac.jp
}

\author{
Adam Van Tuyl \\ Department of Mathematics and Statistics \\ McMaster University \\ Hamilton, ON, L8S 4L8, Canada \\ vantuyl@math.mcmaster.ca
}

Submitted: Oct 17, 2018; Accepted: Feb 9, 2019; Published: Feb 22, 2019

(C) The authors. Released under the CC BY-ND license (International 4.0).

\begin{abstract}
For any two integers $d, r \geqslant 1$, we show that there exists an edge ideal $I(G)$ such that $\operatorname{reg}(R / I(G))$, the Castelnuovo-Mumford regularity of $R / I(G)$, is $r$, and $\operatorname{deg} h_{R / I(G)}(t)$, the degree of the $h$-polynomial of $R / I(G)$, is $d$. Additionally, if $G$ is a graph on $n$ vertices, we show that $\operatorname{reg}(R / I(G))+\operatorname{deg} h_{R / I(G)}(t) \leqslant n$.
\end{abstract}

Mathematics Subject Classifications: 13D02, 13D40, 05C69, 05C70, 05E40

\section{Introduction}

Let $I$ be a homogeneous ideal of the polynomial $\operatorname{ring} R=k\left[x_{1}, \ldots, x_{n}\right]$ where $k$ is a field. Associated to $I$ is a graded minimal free resolution of the form

$$
0 \rightarrow \bigoplus_{j \in \mathbb{N}} R(-j)^{\beta_{p, j}(I)} \rightarrow \cdots \rightarrow \bigoplus_{j \in \mathbb{N}} R(-j)^{\beta_{1, j}(I)} \rightarrow R \rightarrow R / I \rightarrow 0
$$

where $R(-j)$ denotes the polynomial ring $R$ with its grading twisted by $j$, and $\beta_{i, j}(I)$ is the $i, j$-th graded Betti number. This resolution encodes a number of important invariants of $R / I$. One such invariant is the (Castelnuovo-Mumford) regularity of $I$, which is defined by

$$
\operatorname{reg}(R / I)=\max \left\{j-i \mid \beta_{i, j}(I) \neq 0\right\}
$$


The Hilbert series of $R / I$, that is, $H_{R / I}(t)=\sum_{j \in \mathbb{N}} \operatorname{dim}_{k}(R / I)_{j} t^{j}$, can also be read from this resolution; in particular, if $b_{i, i+j}=\beta_{i, i+j}(I)$, then (see $[7$, p. 100])

$$
H_{R / I}(t)=\frac{\sum_{i}(-1)^{i}\left(\sum_{j} b_{i, i+j} t^{i+j}\right)}{(1-t)^{n}} .
$$

This rational function may or may not be in lowest terms; when we rewrite $H_{R / I}(t)$ in lowest terms, the Hilbert-Serre theorem (see [1, Proposition 4.4.1]) says

$$
H_{R / I}(t)=\frac{h_{R / I}(t)}{(1-t)^{\operatorname{dim}(R / I)}} \quad \text { with } h(t) \in \mathbb{Z}[t] \text { and } h(1) \neq 0 .
$$

The polynomial $h_{R / I}$ is called the $h$-polynomial of $R / I$.

Given that $\operatorname{reg}(R / I)$ and $\operatorname{deg} h_{R / I}(t)$ are both derived from the graded minimal free resolution, one can ask if there is any relationship between these two invariants. For example, from [1, Lemma 4.1.3], it follows that if $I$ has a pure resolution (for each $i$, there is at most one $j$ such that $\left.\beta_{i, i+j}(I) \neq 0\right)$, then

$$
\operatorname{deg} h_{R / I}(t)-\operatorname{reg}(R / I)=\operatorname{dim}(R / I)-\operatorname{depth}(R / I) .
$$

The first two authors initiated a comparison of these two invariants in $[9,10,11]$. It was shown in [9] that for all $r, d \geqslant 1$, there exists a monomial ideal such that $\operatorname{reg}(R / I)=r$ and $\operatorname{deg}(R / I)=d$; in [10], it shown that this monomial ideal could be taken to be a lexsegment monomial ideal. In both cases, the degrees of the minimal generators of $I$ depend upon on $r$ and/or $d$. However, if restrict our family of ideals, one might expect some restriction on the values of $r$ and $d$. For example, it is shown in [11] that for $2 \leqslant r \leqslant d$, there exists a binomial edge ideal (see $[8,14]) J_{G}$ with $\operatorname{reg}\left(R / J_{G}\right)=r$ and $\operatorname{deg} h_{R / J_{G}}(t)=d$, and furthermore, [16, Theorem 2.1] says that $\operatorname{deg} h_{R / J_{G}}(t)=1$ if $\operatorname{reg}\left(R / J_{G}\right)=1$.

The starting point of this paper is to ask what happens if we restrict to edge ideals. Recall that if $G=(V(G), E(G))$ is a finite simple graph on $V(G)=\left\{x_{1}, \ldots, x_{n}\right\}$, then the edge ideal is the ideal $I(G)=\left(x_{i} x_{j} \mid\left\{x_{i}, x_{j}\right\} \in E\right) \subseteq R=k\left[x_{1}, \ldots, x_{n}\right]$. Our main result is the perhaps surprising fact that one can obtain the main result of [9] using only edge ideals (unlike $[9,10]$ where the degrees of the generators change, our generators always have degree two):

Theorem 1 (Theorem 4). Let $r, d \geqslant 1$ be integers. Then there is a finite simple graph $G$ with $r=\operatorname{reg}(R / I(G))$ and $d=\operatorname{deg} h_{R / I(G)}(t)$.

Our proof of Theorem 1 uses the following strategy. We show that if $G$ is a graph with $\operatorname{reg}(R / I(G))=r$ and $\operatorname{deg} h_{R / I(G)}(t)=d$, then one can construct a new graph $G^{\prime}$ with $\operatorname{reg}\left(R / I\left(G^{\prime}\right)\right)=r+1$ and $\operatorname{deg} h_{R / I\left(G^{\prime}\right)}(t)=d+1$. The proof of Theorem 1 then reduces to constructing graphs with $\left(\operatorname{reg}(R / I(G)), \operatorname{deg} h_{R / I(G)}(t)\right)=(1, d)$ or $(r, 1)$ for any integers $d, r \geqslant 1$.

Interestingly, reg $(R / I(G))$ and $\operatorname{deg} h_{R / I(G)}(t)$ are related by the following inequality. 
Theorem 2 (Theorem 13). Let $G$ be a graph on $n$ vertices. Then

$$
\operatorname{reg}(R / I(G))+\operatorname{deg} h_{R / I(G)}(t) \leqslant n .
$$

We provide examples to show that this bound is sharp. Note that Theorem 2 gives a new upper bound on the regularity of edge ideals, i.e., $\operatorname{reg}(R / I(G)) \leqslant n-\operatorname{deg} h_{R / I(G)}(t)$, which complements past research on the regularity of edge ideals (see $[5,6])$.

\section{Background}

We recall the relevant graph theory and commutative algebra background. We continue to use the notation and terminology from the introduction.

Let $G=(V(G), E(G))$ be a finite simple graph on the vertex set $V(G)=\left\{x_{1}, \ldots, x_{n}\right\}$ and edge set $E(G)$ consisting of unordered pairs of distinct elements of $V(G)$, that is, if $e \in E(G)$, then $e=\left\{x_{i}, x_{j}\right\}$ for some $i \neq j$. If $G$ is clear, we write $V$, respectively $E$, for $V(G)$, respectively $E(G)$.

We say that there is a path between the vertices $x_{i}$ and $x_{j}$ if there is a collection of edges $\left\{e_{1}, e_{2}, \ldots, e_{t}\right\}$ such that $x_{i} \in e_{1}, x_{j} \in e_{t}$, and $e_{\ell} \cap e_{\ell+1} \neq \varnothing$ for all $\ell=1, \ldots, t-1$. A graph $G$ is connected if there is a path between every pair of vertices of $G$; otherwise, $G$ is said to be disconnected. A connected component of $G$ is a maximal connected subgraph.

Given any subset $W \subseteq V(G)$, the induced subgraph of $G$ on $W$ is the graph $G_{W}=$ $\left(W, E\left(G_{W}\right)\right)$ where $E\left(G_{W}\right)=\{e \in E(G) \mid e \subseteq W\}$. Given an $x \in V(G)$, the set of neighbours of $x$ is the set $N(x)=\{y \mid\{x, y\} \in E(G)\}$.

A set of vertices $W \subseteq V$ is an independent set if for all $e \in E, e \nsubseteq W$. An independent set is a maximal independent set if it is maximal with respect to inclusion. We let $\alpha(G)$ denote the size of the largest maximal independent set. Using the independent sets, we can build a simplicial complex. In particular, the independence complex of $G$ is the simplicial complex:

$$
\operatorname{Ind}(G)=\{W \subseteq V \mid W \text { is an independent set }\} .
$$

Note that $\alpha(G)$ is the cardinality of the largest element in $\operatorname{Ind}(G)$.

A set of vertices $W \subseteq V$ is a vertex cover if for all $e \in E, e \cap W \neq \varnothing$. A vertex cover is a minimal vertex cover if it is minimal with respect to inclusion. We let $\beta(G)$ denote the size of the smallest minimal vertex cover. There is duality between independent sets and vertex covers; specifically, $W \subseteq V$ is an independent set if and only if $V \backslash W$ is a vertex cover. Consequently

$$
\alpha(G)+\beta(G)=n .
$$

A set of edges $\left\{e_{1}, \ldots, e_{s}\right\} \subseteq E$ is said to be a matching if none of the edges share a common vertex. We let $\alpha^{\prime}(G)$ denote the size of the maximum matching in $G$. We then always have the following inequality:

$$
\alpha^{\prime}(G) \leqslant \beta(G)
$$


Indeed, for any matching $\left\{e_{1}, \ldots, e_{s}\right\} \subseteq E$, any minimal vertex cover must contain at least one vertex from each $e_{i}$.

Finally, we will require the following bound on the regularity of $R / I(G)$.

Theorem 3 ([6, Theorem 6.7]). For any finite simple graph $G, \operatorname{reg}(R / I(G)) \leqslant \alpha^{\prime}(G)$.

\section{Main Theorem}

In this section we will prove our main theorem:

Theorem 4. Let $r, d \geqslant 1$ be integers. Then there is a finite simple graph $G$ with $r=$ $\operatorname{reg}(R / I(G))$ and $d=\operatorname{deg} h_{R / I(G)}(t)$.

In order to show this theorem, we will prepare some lemmata.

Lemma 5 ([12, Lemma 3.2]). Let $R_{1}=k\left[x_{1}, \ldots, x_{n^{\prime}}\right]$ and $R_{2}=k\left[x_{n^{\prime}+1}, \ldots, x_{n}\right]$ be polynomial rings over a field $k$. Let $I_{1}$, respectively $I_{2}$, be a nonzero homogeneous ideal of $R_{1}$, respectively $R_{2}$. We write $R$ for $R_{1} \otimes_{k} R_{2}=k\left[x_{1}, \ldots, x_{n}\right]$ and regard $I_{1}+I_{2}$ as a homogeneous ideal of $R$. Then

$$
\begin{aligned}
\operatorname{reg}\left(R / I_{1}+I_{2}\right) & =\operatorname{reg}\left(R_{1} / I_{1}\right)+\operatorname{reg}\left(R_{2} / I_{2}\right), \quad \text { and } \\
H_{R / I_{1}+I_{2}}(t) & =H_{R_{1} / I_{1}}(t) \cdot H_{R_{2} / I_{2}}(t) .
\end{aligned}
$$

By virtue of this lemma, one has:

Lemma 6. Let $G$ be a simple graph, and let $G_{1}, \ldots, G_{\ell}$ be the connected components of G. Then

$$
\operatorname{reg}(R / I(G))=\sum_{i=1}^{\ell} \operatorname{reg}\left(R_{i} / I\left(G_{i}\right)\right), \quad \text { and } \operatorname{deg} h_{R / I(G)}(t)=\sum_{i=1}^{\ell} \operatorname{deg} h_{R_{i} / I\left(G_{i}\right)}(t),
$$

where $R_{i}=k\left[x_{j} \mid j \in V\left(G_{i}\right)\right]$ for $i=1, \ldots, \ell$, and $R=R_{1} \otimes_{k} \cdots \otimes_{k} R_{\ell}$.

Remark 7. By Lemma 6, if $G$ is graph with $\operatorname{reg}(R / I(G))=r$ and $\operatorname{deg} h_{R / I(G)}(t)=d$, then the graph $G^{\prime}$ which is the disjoint union of $G$ and a single edge on two new vertices $\left\{z_{1}, z_{2}\right\}$ has $\operatorname{reg}\left(R^{\prime} / I\left(G^{\prime}\right)\right)=r+1$ and $\operatorname{deg} h_{R^{\prime} / I\left(G^{\prime}\right)}(t)=d+1$ where $R^{\prime}=R \otimes_{k} k\left[z_{1}, z_{2}\right]$. To prove Theorem 4 we need to show that for each $r \geqslant 1$, there exists a graph $G$ with $\operatorname{reg}(R / I(G))=r$ and $\operatorname{deg} h_{R / I(G)}(t)=1$, and for each $d \geqslant 1$, there is a graph $G$ with $\operatorname{reg}(R / I(G))=1$ and $\operatorname{deg} h_{R / I(G)}(t)=d$. We now work towards this goal.

Example 8. Let $d \geqslant 1$ be a positive integer and let $K_{d, d}$ be the complete bipartite graph, i.e., the graph with $V\left(K_{d, d}\right)=\left\{x_{1}, \ldots, x_{d}, y_{1}, \ldots, y_{d}\right\}$ and $E\left(K_{d, d}\right)=\left\{x_{i} y_{j} \mid 1 \leqslant i, j \leqslant d\right\}$. By virtue of Fröberg's Theorem [3, Theorem 1], one has $\operatorname{reg}\left(R / I\left(K_{d, d}\right)\right)=1$. In addition, the Hilbert series of $R / I\left(K_{d, d}\right)$ can be computed from the graded minimal free resolution (e.g., see [13, Theorem 5.2.4]); in particular:

$$
H_{R / I\left(K_{d, d}\right)}(t)=\frac{-(1-t)^{d}+2}{(1-t)^{d}} .
$$

Hence $\operatorname{deg} h_{R / I\left(K_{d, d}\right)}(t)=d$. 
We now require the following graph construction. Let $G$ be a simple graph on $V(G)=$ $\left\{x_{1}, \ldots, x_{n}\right\}$. For $S \subset V(G)$, the graph $G^{S}$ is defined by

- $V\left(G^{S}\right)=V(G) \cup\left\{x_{n+1}\right\}$, where $x_{n+1}$ is a new vertex; and

- $E\left(G^{S}\right)=E(G) \cup\left\{\left\{x_{i}, x_{n+1}\right\} \mid x_{i} \in S\right\}$.

Lemma 9. Let $G$ be a graph and let $S \subset V(G)$. Assume that

- $\operatorname{dim} R / I(G) \geqslant 2$ and $h_{R / I(G)}(t)=1+h_{1} t+h_{2} t^{2}$;

- $\operatorname{reg}(R / I(G)) \geqslant 2$;

- $|S|=|V(G)|-\operatorname{dim} R / I(G)+2$; and

- For any $u \in V(G) \backslash S$, there exists $u^{\prime} \in S$ such that $\left\{u, u^{\prime}\right\} \in E(G)$.

Then

$$
H_{R^{\prime} / I\left(G^{S}\right)}(t)=\frac{1+\left(h_{1}+1\right) t+\left(h_{2}-1\right) t^{2}}{(1-t)^{\operatorname{dim} R / I(G)}} \text { and } \operatorname{reg}\left(R^{\prime} / I\left(G^{S}\right)\right)=r
$$

where $R^{\prime}=R \otimes_{k} k\left[x_{n+1}\right]$.

Proof. By the assumptions and the definition of $G^{S}$, we have $I\left(G^{S}\right)+\left(x_{n+1}\right)=\left(x_{n+1}\right)+$ $I(G)$, and $I\left(G^{S}\right):\left(x_{n+1}\right)=\left(x_{i} \mid x_{i} \in S\right)$. Hence $R^{\prime} /\left(I\left(G^{S}\right)+\left(x_{n+1}\right)\right) \cong R / I(G)$ and $R^{\prime} /\left(I\left(G^{S}\right):\left(x_{n+1}\right)\right) \cong k\left[x_{i} \mid x_{i} \notin S\right] \otimes_{k} k\left[x_{n+1}\right]$. Thus, by the additivity of Hilbert series on the short exact sequence

$$
0 \rightarrow\left(R^{\prime} /\left(I\left(G^{S}\right):\left(x_{n+1}\right)\right)\right)(-1) \stackrel{\times x_{n+1}}{\longrightarrow} R^{\prime} / I\left(G^{S}\right) \rightarrow R^{\prime} /\left(I\left(G^{S}\right)+\left(x_{n+1}\right)\right) \rightarrow 0,
$$

we have

$$
\begin{aligned}
H_{R^{\prime} / I\left(G^{S}\right)}(t) & =H_{R^{\prime} /\left(I\left(G^{S}\right)+\left(x_{n+1}\right)\right)}(t)+t \cdot H_{R^{\prime} /\left(I\left(G^{S}\right):\left(x_{n+1}\right)\right)}(t) \\
& =H_{R / I(G)}(t)+\frac{t}{(1-t)^{|V(G)|-|S|+1}} \\
& =\frac{1+h_{1} t+h_{2} t^{2}}{(1-t)^{\operatorname{dim} R / I(G)}}+\frac{t}{(1-t)^{\operatorname{dim} R / I(G)-1}} \\
& =\frac{1+\left(h_{1}+1\right) t+\left(h_{2}-1\right) t^{2}}{(1-t)^{\operatorname{dim} R / I(G)}} .
\end{aligned}
$$

Furthermore, we have reg $\left(R^{\prime} / I\left(G^{S}\right)\right)=r$ by virtue of [2, Lemma 2.10].

Example 10. Let $G$ be the two disjoint edges $\left\{x_{1}, x_{2}\right\}$ and $\left\{x_{3}, x_{4}\right\}$ and $S=V(G)$. Then $G^{S}=G_{\text {ribbon }}$ where $G_{\text {ribbon }}$ is the following graph:

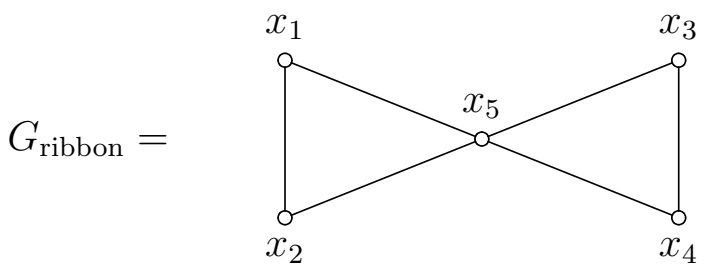


Since $I(G)=\left(x_{1} x_{2}, x_{3} x_{4}\right)$ is a complete intersection, we have $H_{R / I(G)}(t)=\frac{1+2 t+t^{2}}{(1-t)^{2}}$ and $\operatorname{reg}(R / I(G))=2$. Hence, by applying Lemma 9, one has

$$
H_{R^{\prime} / I\left(G_{\text {ribbon }}\right)}(t)=\frac{1+3 t}{(1-t)^{2}} \text { and } \operatorname{reg}\left(R^{\prime} / I\left(G_{\text {ribbon }}\right)\right)=2 .
$$

So $\operatorname{deg} h_{R^{\prime} / I\left(G_{\text {ribbon }}\right)}(t)=1$.

Example 11. Let $G_{0}$ be the union of $G_{\text {ribbon }}$ and a disjoint edge $\left\{x_{6}, x_{7}\right\}$ :

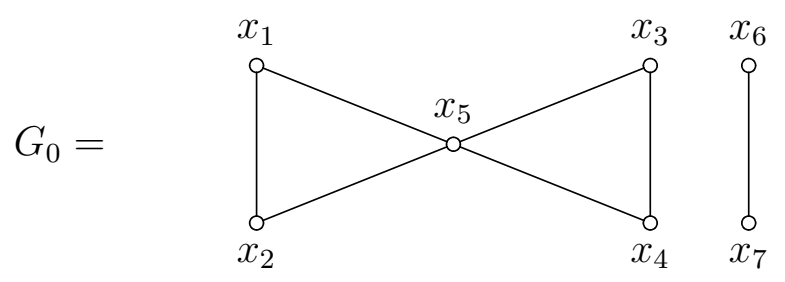

Then $H_{R / I\left(G_{0}\right)}(t)=\frac{1+3 t}{(1-t)^{2}} \cdot \frac{1+t}{1-t}=\frac{1+4 t+3 t^{2}}{(1-t)^{3}}$ and $\operatorname{reg}\left(R / I\left(G_{0}\right)\right)=2+1=3$ by virtue of Lemma 6 and Example 10. Now we set $S_{i}=V\left(G_{i}\right) \backslash\left\{x_{7}\right\}$ and $G_{i+1}=G_{i}^{S_{i}}$ for $i=0,1,2$. Then, by using Lemma 9 repeatedly, one has

$$
H_{R^{\prime} / I\left(G_{3}\right)}(t)=\frac{1+7 t}{(1-t)^{3}} \text { and } \operatorname{reg}\left(R^{\prime} / I\left(G_{3}\right)\right)=3,
$$

where $R^{\prime}=k\left[x_{1}, \ldots, x_{10}\right]$ and $G_{3}$ is the following graph:

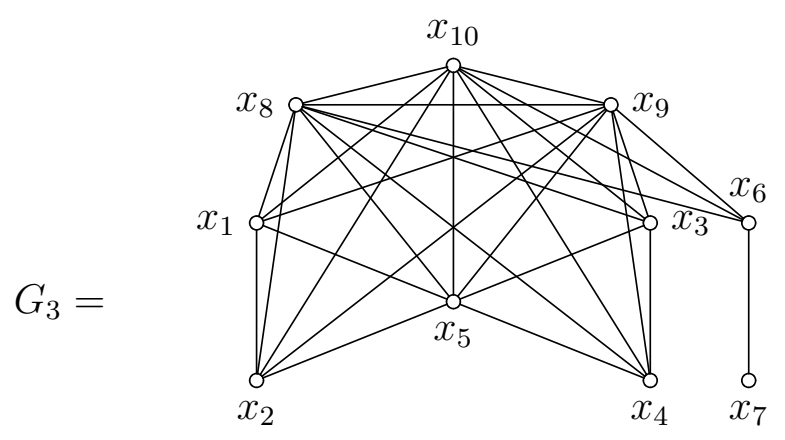

Lemma 9 says that, given $r \geqslant 2$, we can construct a graph $G^{\prime}$ with $\operatorname{deg} h_{R / I\left(G^{\prime}\right)}(t)=1$ and $\operatorname{reg}\left(R / I\left(G^{\prime}\right)\right)=r$ from a graph $G$ for which $\operatorname{deg} h_{R / I(G)}(t)=2$ and $\operatorname{reg}(R / I(G))=r$, provided the hypotheses of Lemma 9 are met. We use this idea in the next lemma.

Lemma 12. Given an integer $r \geqslant 3$, we put

$$
Y_{r}=\left\{y_{1,1}, y_{2,1} \ldots, y_{r-2,1}, y_{1,2}, y_{2,2}, \ldots, y_{r-2,2}\right\},
$$




$$
Z_{r}=\bigcup_{i=1}^{r-2}\left\{z_{1}^{(i)}, z_{2}^{(i)}, \ldots, z_{2^{i+1}-1}^{(i)}\right\}
$$

and

$$
X=\left\{x_{1}, x_{2}, x_{3}, x_{4}, x_{5}\right\}
$$

Let $G^{(r)}$ be the graph on $X \cup Y_{r} \cup Z_{r}$ such that

- the induced subgraph $G_{X, Y_{r}}^{(r)}$ is the following:

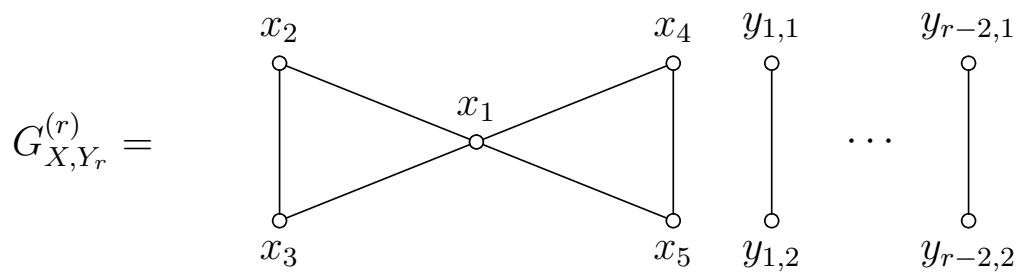

- the induced subgraph $G_{Z_{r}}^{(r)}$ is a complete graph, i.e., all vertices are adjacent; and

- for all $1 \leqslant i \leqslant r-2$ and for all $1 \leqslant j \leqslant 2^{i+1}-1$,

$$
N_{G}\left(z_{j}^{(i)}\right)=X \cup\left\{y_{1,1}, y_{2,1}, \ldots, y_{r-2,1}\right\} \cup Z_{r} \backslash\left\{z_{j}^{(i)}\right\} .
$$

Let $R^{(r)}=k\left[\left\{X \cup Y_{r} \cup Z_{r}\right\}\right]$ be the polynomial ring over $k$ whose variables equal to $X \cup$ $Y_{r} \cup Z_{r}$. Then

$$
\begin{aligned}
& \text { 1. } H_{R^{(r)} / I\left(G^{(r)}\right)}(t)=\frac{1+\left(2^{r}-1\right) t}{(1-t)^{r}} \text {, that is, } \operatorname{deg} h_{R^{(r)} / I\left(G^{(r)}\right)}(t)=1 \text {, and } \\
& \text { 2. } \operatorname{reg}\left(R^{(r)} / I\left(G^{(r)}\right)\right)=r \text {. }
\end{aligned}
$$

Proof. We prove this lemma by induction on $r \geqslant 3$. The graph of Example 11 is $G^{(3)}$; we showed that $H_{R^{(3)} / I\left(G^{(3)}\right)}(t)=\frac{1+7 t}{(1-t)^{3}}$ and $\operatorname{reg}\left(R^{(3)} / I\left(G^{(3)}\right)\right)=3$.

Assume $r>3$. Let $G^{\prime}$ be the union of $G^{(r-1)}$ and a disjoint edge $\left\{y_{r-2,1}, y_{r-2,2}\right\}$. Let $R^{\prime}=R^{(r-1)} \otimes_{k} k\left[y_{r-2,1}, y_{r-2,2}\right]$. Then

$$
\begin{aligned}
H_{R^{\prime} / I\left(G^{\prime}\right)}(t) & =H_{R^{r-1} / I\left(G^{(r-1)}\right)}(t) \cdot \frac{1+t}{1-t}=\frac{1+\left(2^{r-1}-1\right) t}{(1-t)^{r-1}} \cdot \frac{1+t}{1-t} \\
& =\frac{1+2^{r-1} t+\left(2^{r-1}-1\right) t^{2}}{(1-t)^{r}}
\end{aligned}
$$

and

$$
\operatorname{reg}\left(R^{\prime} / I\left(G^{\prime}\right)\right)=\operatorname{reg}\left(R^{(r-1)} / I\left(G^{(r-1)}\right)\right)+1=r-1+1=r
$$

by the induction hypothesis and Lemma 9 . 
Let $S_{0}=X \cup\left\{y_{1,1}, y_{1,2}, \ldots, y_{r-2,1}\right\} \cup Z_{r-1}$. Then $\left|S_{0}\right|=r+3+\left|Z_{r-1}\right|$ and

$$
\begin{aligned}
\left|V\left(G^{\prime}\right)\right|-\operatorname{dim} R^{\prime} / I\left(G^{\prime}\right)+2 & =|X|+\left|Y_{r-1}\right|+\left|Z_{r-1}\right|+2-r+2 \\
& =5+2(r-3)+\left|Z_{r-1}\right|+4-r \\
& =r+3+\left|Z_{r-1}\right| .
\end{aligned}
$$

Hence, by virtue of Lemma 9, one has

$$
H_{R_{0} / I\left(G_{0}\right)}(t)=\frac{1+\left(2^{r-1}+1\right) t+\left(2^{r-1}-2\right) t^{2}}{(1-t)^{r}} \text { and } \operatorname{reg}\left(R_{0} / I\left(G_{0}\right)\right)=r,
$$

where $R_{0}=R^{\prime} \otimes_{k} k\left[z_{1}^{(r-2)}\right], G_{0}=\left(G^{\prime}\right)^{S_{0}}$, and $V\left(G_{0}\right)=V\left(G^{\prime}\right) \cup\left\{z_{1}^{(r-2)}\right\}$.

Now, for each $1 \leqslant j \leqslant 2^{r-1}-2$, we define $R_{j}, S_{j}$ and $G_{j}$ inductively:

- $R_{j}=R_{j-1} \otimes_{k} k\left[z_{j+1}^{(r-2)}\right]$;

- $S_{j}=S_{j-1} \cup\left\{z_{j+1}^{(r-2)}\right\}$; and

- $G_{j}=G_{j-1}^{S_{j}}$.

Then $R_{2^{r-1}-2}=R^{(r)}, G_{2^{r-1}-2}=G^{(r)}$, and one has

$$
H_{R^{(r)} / I\left(G^{(r)}\right)}(t)=\frac{1+\left(2^{r}-1\right) t}{(1-t)^{r}} \text { and } \operatorname{reg}\left(R^{(r)} / I\left(G^{(r)}\right)\right)=r
$$

by using Lemma 9 repeatedly.

We are now in a position to finish the proof of Theorem 4.

Proof (of Theorem 4). We discuss each of the following three cases.

Case 1. Suppose that $1 \leqslant r \leqslant d$. Let $G$ be the union of $K_{d-r+1, d-r+1}$ and $(r-1)$ disjoint edges. By virtue of Lemma 6 and Example 8, one has

$$
\operatorname{reg}(R / I(G))=1+(r-1)=r \quad \text { and } \quad \operatorname{deg} h_{R / I(G)}(t)=(d-r+1)+(r-1)=d .
$$

Case 2. Suppose that $r, d \geqslant 1$ are integers with $r-d=1$. Let $G$ be the union of $G_{\text {ribbon }}$ and $(r-2)$ disjoint edges. By virtue of Lemma 6 and Example 10, one has

$$
\operatorname{reg}(R / I(G))=2+(r-2)=r, \quad \text { and } \quad \operatorname{deg} h_{R / I(G)}(t)=1+(r-2)=r-1=d .
$$

Case 3. Suppose that $r, d \geqslant 1$ are integers with $r-d>1$. Let $G$ be the union of the graph $G^{(r-d+1)}$ of Lemma 12 and $(d-1)$ disjoint edges. By virtue of Lemma 6 and 12, one has

$$
\operatorname{reg}(R / I(G))=(r-d+1)+(d-1)=r, \quad \text { and } \quad \operatorname{deg} h_{R / I(G)}(t)=1+(d-1)=d .
$$




\section{Comparing the regularity and $h$-polynomial for fixed $n$}

Theorem 4 shows that for all $(r, d) \in \mathbb{N}_{\geqslant 1}^{2}$, there exists a finite simple graph $G$ with $\left(\operatorname{reg}(R / I(G)), \operatorname{deg} h_{R / I(G)}(t)\right)=(r, d)$. However, if we fix $n=|V(G)|$, then the regularity of $R / I(G)$ and the degree of the $h$-polynomial must also satisfy the following inequality:

Theorem 13. Let $G$ be a finite simple graph on $n$ vertices. Then

$$
\operatorname{deg} h_{R / I(G)}(t)+\operatorname{reg}(R / I(G)) \leqslant n .
$$

Proof. Via the Stanley-Reisner correspondence, the edge ideal $I(G)$ is associated to the independence complex $\operatorname{Ind}(G)$. The Hilbert series of $R / I(G)$ can then be expressed as

$$
H_{R / I(G)}(t)=\sum_{i=0}^{d} \frac{f_{i-1} t^{i}}{(1-t)^{i}}
$$

(see [7, Theorem 6.2.1]) where $f_{j-1}$ is the number of independent sets of cardinality $j$ in $G$ (in other words, this in the number of faces of $\operatorname{Ind}(G)$ of dimension $j-1$ ). In particular, $d=\alpha(G)$, the size of the largest independent set. It follows that $\operatorname{deg} h_{R / I(G)}(t) \leqslant \alpha(G)$. By combining Theorem 3 and the inequality (2), we have the bound $\operatorname{reg}(R / I(G)) \leqslant$ $\alpha^{\prime}(G) \leqslant \beta(G)$. Thus

$$
\operatorname{deg} h_{R / I(G)}(t)+\operatorname{reg}(R / I(G)) \leqslant \alpha(G)+\beta(G)=n,
$$

as desired, where the last equality is (1).

Remark 14. For an alternative proof, [15, Corollary 4.3] can be used to show that $\operatorname{deg} h_{R / I(G)}(t) \leqslant(n-\beta(G))$.

Example 15. The upper bound of Theorem 13 is sharp. In fact, we can give two families of graphs such that the equality $\operatorname{deg} h_{R / I(G)}(t)+\operatorname{reg}(R / I(G))=n$ holds. For the first family, let $n=2 m$ and let $G$ be the union of $m$ disjoint edges. Then $\operatorname{deg} h_{R / I(G)}(t)=$ $\operatorname{reg}(R / I(G))=m$. For the second family, let $G=K_{1, n-1}$ be the star graph. Then $\operatorname{deg} h_{R / I(G)}(t)=n-1$ and $\operatorname{reg}(R / I(G))=1$.

Remark 16. We end with an observation based upon our computer experiments. For any graph $G$ with at least one edge, we have $\operatorname{reg}(R / I(G)) \geqslant 1$ and $\operatorname{deg}(R / I(G)) \geqslant 1$. If we fix an $n=|V(G)|$, it is natural to ask if we can describe all pairs $(r, d) \in \mathbb{N}_{\geqslant 1}^{2}$ for which there is a graph $G$ on $n$ vertices with $r=\operatorname{reg}(R / I(G))$ and $d=\operatorname{deg} h_{R / I(G)}(t)$. Theorem 13 implies that $r+d \leqslant n$. Furthermore, note that $\alpha^{\prime}(G) \leqslant\left\lfloor\frac{n}{2}\right\rfloor$, so we must also have $r \leqslant\left\lfloor\frac{n}{2}\right\rfloor$ by Theorem 3 .

However, these inequalities are not enough to desribe all the pairs $(r, d)$ that may be realizable. For example, when $n=9$, we computed $\left(\operatorname{reg}(R / I(G))\right.$, deg $\left.h_{R / I(G)}(t)\right)$ for all 274668 graphs on nine vertices. We observed that for all such $G$,

$$
\left(\operatorname{reg}(R / I(G)), \operatorname{deg} h_{R / I(G)}(t)\right) \notin\{(3,1),(4,1),(4,2)\}
$$

even though these tuples satisfy the inequalities $r+d \leqslant 9$ and $r \leqslant 4$. A similar phenomenon was observed for other $n$, thus suggesting the existence of another bound relating $\operatorname{reg}(R / I(G))$ and $\operatorname{deg} h_{R / I(G)}(t)$ for a fixed $n$. 


\section{Acknowledgments.}

The first and last author began discussions on this project at the BIRS (Banff International Research Station) workshop entitled New Trends in Syzygies, organized by Giulio Caviglia and Jason McCullough and held in June 2018. We thank the organizers and BIRS for providing a stimulating research environment. Experiments with Macaulay2 [4] led to many of our results. Hibi and Matsuda's research was supported by JSPS KAKENHI 26220701 and 17K14165. Van Tuyl's research was supported by NSERC Discovery Grant 2014-03898. This work was also made possible by the facilities of the Shared Hierarchical Academic Research Computing Network (SHARCNET: www.sharcnet.ca) and Compute/Calcul Canada.

\section{References}

[1] W. Bruns, J. Herzog, Cohen-Macaulay rings (Revised Edition). Cambridge University Press, 1998.

[2] H. Dao, C. Huneke, J. Schweig, Bounds on the regularity and projective dimension of ideals associated to graphs. J. Algebraic Combin. 38 (2013), 37-55.

[3] R. Fröberg, On Stanley-Reisner rings. Topics in algebra, Banach Center Publications, 26 (1990), 57-70.

[4] D. Grayson, M. Stillman, Macaulay2, a software system for research in algebraic geometry. Available at http://www.math.uiuc.edu/Macaulay2/

[5] H.T. Hà, Regularity of squarefree monomial ideals. Connections between algebra, combinatorics, and geometry, 251-276, Springer Proc. Math. Stat., 76, Springer, New York, 2014.

[6] H.T. Hà, A. Van Tuyl, Monomial ideals, edge ideals of hypergraphs, and their graded Betti numbers. J. Algebraic Combin. 27 (2008), 215-245.

[7] J. Herzog and T. Hibi, Monomial ideals. Graduate Texts in Mathematics 260, Springer, London, 2010.

[8] J. Herzog, T. Hibi, F. Hreindóttir, T. Kahle and J. Rauh, Binomial edge ideals and conditional independence statements. Adv. in Appl. Math. 45 (2010), 317-333.

[9] T. Hibi, K. Matsuda, Regularity and h-polynomials of monomial ideals. Math. Nachr. 291 (2018), 2427-2434.

[10] T. Hibi, K. Matsuda, Lexsegment ideals and their h-polynomials. To appear Acta Math. Vietnam. (2018). arXiv: 1807.02834

[11] T. Hibi, K. Matsuda, Regularity and h-polynomials of binomial edge ideals. Preprint (2018). arXiv:1808.06984

[12] L. T. Hoa, N. D. Tam, On some invariants of a mixed product of ideals. Arch. Math. (Basel) 94 (2010), 327-337.

[13] S. Jacques, Betti numbers of graph ideals. Ph.D. Thesis, University of Sheffield, 2004. arXiv:math/0410107 
[14] M. Ohtani, Graphs and ideals generated by some 2-minors. Comm. Algebra 39 (2011), 905-917.

[15] P. Renteln, The Hilbert series of the face ring of a flag complex. Graphs Combin. 18 (2002), 605-619.

[16] S. Saeedi Madani and D. Kiani, Binomial edge ideals of graphs. Electron. J. Combin. 19 (2012), Paper 44, 6pp. 